\title{
Evaluation of Genetic Diversity in Cotton (Gossypium barbadense L.) Germplasm for Yield and Fibre Attributes by Principle Component Analysis
}

\author{
N. Kumari Vinodhana* and P. Gunasekaran \\ Tamil Nadu Agricultural University, Coimbatore-03, India \\ *Corresponding author
}

\begin{tabular}{|c|}
\hline Keywords \\
\hline $\begin{array}{l}\text { Principle } \\
\text { component analysis, } \\
\text { Skewness, Kurtosis, } \\
\text { Eigen value, } \\
\text { Cumulative } \\
\text { variability }\end{array}$ \\
\hline Article Info \\
\hline $\begin{array}{l}\text { Accepted: } \\
20 \text { March } 2019 \\
\text { Available Online: } \\
10 \text { April } 2019\end{array}$ \\
\hline
\end{tabular}

\section{A B S T R A C T}

Fifty cotton genotypes were investigated for variability and genetic divergence regarding yield related attributes, seed cotton yield and fibre quality traits using principle component analysis. Eleven traits viz., days to flowering, plant height $(\mathrm{cm})$, no of sympodia/plant, no of bolls, boll weight (g/plant), seed cotton yield (kg/ha), Micronnaire value, \% Span length, Uniformity ratio, Bundle strength (g/tex) and Elongation ratio were recorded for the germplasm accessions. Maximum variation of about $17.0 \% \mathrm{CV}$ was observed for the traits No of bolls, Micronnaire value and Elongation ratio followed by Plant height (16.4) and Seed cotton yield (15.23). The study of distribution of quantitative traits using skewness and kurtosis provides information about nature of gene action and number of genes controlling the traits respectively. The traits No of sympodia, No of bolls, Seed cotton yield, Micronnaire value, Bundle strength, Elongation ratio were found to possess positive skewness resulting in complementary gene interactions. Regarding kurtosis, the traits No of bolls, boll weight and seed cotton yield were exhibited a normal distribution. Principal component analysis was utilized to examine the variation and to estimate the relative contribution of various traits for total variability. In the current study, Out of Eleven principle components (PCs), five principle components revealed Eigenvalue $>1$ and $76.0 \%$ of cumulative variability for the attributes under examination. The PC1 and PC2 contributed towards $42 \%$ of cumulative variability. The attributes of significance depicted in PC I and PC II were seed cotton yield, no of bolls, no of sympodia, boll weight, micronnaire value and span length expressed great contribution towards cumulative variability. These attributes should have bestowed special emphasis for cotton improvement in future breeding program.

\section{Introduction}

Cotton referred as "White gold" is a premier cash and fibre crop. Four kinds of cotton are cultivated to supply worlds' textile fiber and are vital sources of oil and cottonseed meal. Cultivated species include two diploids $G$. herbaceum and G. arboreum, and two New World tetraploids species, $G$. hirsutum and $G$. barbadense. Gossypium barbedense is known for its better fibre properties being cultivated in less than $2 \%$ in the world. The basic principle of cotton breeding is continuous improvement in genetics of the available plant 
germplasm for creation of new genetic recombinant with objectives of seed cotton yield potential per unit area having acceptable fibre quality under varying agro-climatic conditions.

Cotton production either in seed yield or lint depends on characters like plant height, direct fruit bearing branches (sympodial) and indirect fruit bearing branches (monopodial), boll weight (BW), bolls per plant, seed index, and ginning out turn (Salahuddin et al., 2010). Seed cotton yield is a resultant product of all its component traits and it could be enhanced by exploiting positive influence of yield components. Comprehensive understanding about the crop nature, performance level and association of numerous agronomic attributes with yield is necessary for plant researcher to tackle the cotton yield limiting constraints.

Multivariate biometrical techniques like principle component analysis (PCA), Correlation Analysis and Clustering method have been frequently used to explore genetic diversity among genotypes and its direct and indirect effects (Brown-Guedira et al., 2000). Genetic variation of morphological traits estimated through principal component analysis has led to the recognition of phenotypic variability. Keeping in view the significance of genetic diversity, the present research was conducted in $G$. barbadense genotypes to explore the variability and genetic divergence among cotton germplasm for yield and fibre quality attributes.

\section{Materials and Methods}

A field experiment was carried out at Department of Cotton, Tamil Nadu Agricultural University, Coimbatore. The germplasm collection consisting of 50 cotton accessions used in this study were measured for six quantitative characters viz., days to flowering, plant height $(\mathrm{cm})$, no of sympodia/plant, no of bolls, boll weight (g/plant), seed cotton yield ( $\mathrm{kg} / \mathrm{ha})$ and five fibre quality traits like Micronnaire value, \% Span length, Uniformity ratio, Bundle strength (g/tex) and Elongation ratio. The observations recorded on eleven traits were analyzed in STAR statistical package to explore the genetic divergence through multivariate analysis among cotton germplasm for yield and fibre quality attributes. The PCA analysis reduces the dimensions of a multivariate data to a few principal axes, generates an Eigen vector for each axis and produces component scores for the characters.

\section{Results and Discussion}

The first order statistics for the variables measured for 50 cotton germplasm accessions are given in the Table 1. Maximum variation of about $17.0 \% \mathrm{CV}$ was observed for the traits No of bolls, Micronnaire value and Elongation ratio followed by Plant height (16.4), Seed cotton yield (15.23), Bundle strength (12.77) and No of Sympodia (12.57). Days to flowering has shown the least variation with the CV\% of 3.44 .

Among the 50 genotypes, CCB 4, Seabrook and NDGB 49 was observed to be early flowering in about 63 days and NDGB 60 was found to late flowering with the mean flowering duration of 67 days. This trait showed negative skewness (-0.17) and kurtosis (-0.76). ICB 192 had the tall plant height of $136.30 \mathrm{~cm}$ and the genotype NDGB 76 had the shortest plant type $(63.3 \mathrm{~cm})$ with the mean value of $101.00 \mathrm{~cm}$. This trait showed negative skewness (-0.17) and kurtosis (-0.76).

The number of Sympodia was found to be more in ICB 100 (28.0) and less in ICB 134 (15.7) with the overall mean of 21.36. The trait showed the positive skewness (0.38) and 
negative kurtosis (-0.05). The genotype NDGB 33 found to have maximum number of bolls (33.3) and ICB 367 had less no of bolls (18.7). The mean number of bolls of the genotypes was 23.45. The trait showed positive skewness (1.14) and kurtosis (0.21).

Boll weight was found to be higher in ICB 365 (4.5) and lower in ICB 122 (3.3) with the mean weight of $3.9 \mathrm{~g}$. The trait showed negative skewness (-0.43) and positive kurtosis (0.12). Seed cotton yield was found to be highest in the genotype NDGB 28 (989.80) followed by NDGB 33 (976.5) and NDGB 60 (935.6) and found to be lowest in ICB 227 (570.4). The mean value of the genotypes for seed cotton yield was 723.05 kg.ha. The skewness and kurtosis of the trait are in positive direction.

NDGB 28 had the highest micronnaire value of 6.8 while the genotype CCB4 had lowest micronnaire value of 2.8 with the mean value of 3.9. The trait had positive skewness (1.6) and kurtosis (4.63). The genotype ICB 124 was found to have maximum Uniformity Ratio (58.5) and Bundle strength (29.5) while the genotypes ICB 204 (35.8) and ICB 188 (18.2) had the minimum value for the respective fibre quality traits. The overall mean of the genotypes for uniformity ratio and bundle strength was 50.96 and 22.83 respectively. The trait Uniformity Ratio had negative skewness (-1.11) and positive kurtosis (4.63) while bundle strength had positive skewness (0.38) and negative kurtosis (052). The genotype CCB 4 had the maximum Span length of 38.0 and the genotype ICB20 had the minimum span length of 24.3 with the mean value of 30.6. Span length exhibited negative skewness and positive kurtosis. The elongation ratio was found to be highest in TCB 26 (4.90) and lowest in CCB 4 (1.48). Both the skewness and kurtosis are in positive direction.
Frequency distribution for different traits on 50 germplasm accessions revealed different patterns of distribution as shown on Figure 1.

The study of distribution of quantitative traits using skewness and kurtosis provides information about nature of gene action and number of genes controlling the traits respectively. The skewed distribution of a trait in general suggests that the trait is under the control of non-additive gene action and is influenced by environmental variables. Positive skewness is associated with complementary gene interactions while negative skewness is associated with duplicate (additive $\mathrm{x}$ additive) gene interactions.

In this study, the traits No of sympodia, No of bolls, Seed cotton yield, Micronnaire value, Bundle strength, Elongation ratio were found to possess positive skewness while the traits days to flowering, plant height, boll weight, span length found to have negative skewness. The genes controlling the trait with skewed distribution tend to be predominantly dominant irrespective of whether they have increasing or decreasing effect on the trait.

Kurtosis is important because it affects the measure of dispersion we use to describe the data in the distribution. In a platykurtic or flat distribution, the variance and standard deviation will be greater than in a normal or leptokurtic distribution; this means that there is more dispersion or variability in a platykurtic distribution than in either of the other shapes. In a leptokurtic distribution or peaked distribution, the variance and standard deviation will be less than in a normal or platykurtic distribution; this means that there is less dispersion or variability in a leptokurtic distribution than in either of the other shapes.

Kurtosis is negative or close to zero in the absence of gene interaction and is positive in 
the presence of gene interactions. The traits with leptokurtic and platykurtic distribution are controlled by fewer and large number of genes, respectively. In this study, the traits days to flowering, plant height, no of sympodia, and bundle strength were found to be platykurtic while the traits micronnaire value, span length, uniformity ratio and elongation ratio exhibited leptokurtic distribution. The traits No of bolls, boll weight and seed cotton yield were found to exhibit a normal distribution where the variance and standard deviation will be between those figures for a platykurtic or leptokurtic distribution.

\section{Principle component analysis}

To explore the momentous variation among fifty $G$. barbadense germplasm lines, principle component analysis was used on collected mean data of fibre quality, yield and yield related attributes simultaneously. Principal Component Analysis measures the importance and contribution of each component to total variance. It can be used for measurement of independent impact of a particular trait to the total variance whereas each coefficient of proper vectors indicates the degree of contribution of every original variable with which each principal component is associated. The higher the coefficients, regardless of the sign, the more effective they will be in discriminating between accessions.

In the current study, Out of Eleven principle components (PCs), five principle components revealed Eigenvalue $>1$ and $76.0 \%$ (Figure 1) of cumulative variability for the attributes under examination. The PC I depicted 2.83 Eigen value and $26.0 \%$ variability. The germplasm in PC I exhibited positive effects for seed cotton yield (0.512), no of bolls (0.507), no of sympodia (0.378), micronnaire value (0.337), uniformity ratio (0.296), days to flowering (0.195), Plant height (0.186), bundle strength (0.130), elongation ratio (0.159) and boll weight (0.108) while negative effects was observed for span length (-0.03). The PC II revealed Eigen value of 1.8 Eigenvalue and $16.0 \%$ of variability. The germplasm in PC II showed positive values for span length (0.5757), seed cotton yield (0.249), no of bolls (0.258), no of sympodia (0.105), boll weight (0.024) while other traits showed negative value. The PC III depicted 1.44 Eigen value and $13.0 \%$ of variability. The germplasm in PC III presented positive results only for the traits micronnaire value (0.400), boll weight (0.228) and seed cotton yield (0.053). The PC IV revealed 1.23 Eigen value and $11.0 \%$ of variability.

The germplasm in PC IV exhibited positive effects for seed cotton yield (0.193), boll weight (0.646), micronnaire value (0.066), days to flowering (0.124) and uniformity raitio (0.044). The PC V depicted 1.03 Eigen value and $9.00 \%$ of variability. The germplasm in PC $\mathrm{V}$ presented positive values for boll weight (0.391), Micronaire value (0.068), span length (0.270) and plant height (0.305) while other traits had the negative values (Table 2).

The PC1 and PC2 contributed towards $42 \%$ of cumulative variability. The attributes of significance depicted in PC I and PC II were seed cotton yield, number of bolls, number of sympodia, boll weight, micronnaire value and span length expressed great contribution towards cumulative variability.

These attributes should have bestowed special emphasis for cotton improvement in future breeding program. Previous findings of these attributes depicting their contribution towards cumulative variability and future cotton improvement programs were also reported by Saeed et al., (2014), Kaleri et al., (2015), Latif et al., (2015) and Shah et al., (2018). 
Table.1 Characteristic Means and Variations for 50 Cotton Accessions

\begin{tabular}{|c|c|c|c|c|c|c|c|}
\hline Variable & Mean & Min & Accessions & Max & Accessions & StdDev & $\mathrm{CV}$ \\
\hline DTF & 67.32 & 63 & $\begin{array}{l}\text { CCB 4, } \\
\text { Seabrook, } \\
\text { NDGB } 49\end{array}$ & 72 & NDGB 60 & 2.32 & 3.44 \\
\hline PH (cm) & 100.9 & 63.3 & NDGB 76 & 136.3 & ICB 192 & 16.55 & 16.4 \\
\hline SYM & 21.36 & 15.7 & ICB 134 & 28.0 & ICB 100 & 2.68 & 12.57 \\
\hline NB & 23.45 & 18.7 & ICB 367 & 33.3 & NDGB 33 & 4.02 & 17.14 \\
\hline BW (g) & 3.9 & 3.3 & ICB 122 & 4.5 & ICB 365 & 0.25 & 6.41 \\
\hline SCY (kg/ha) & 723.05 & 570.4 & ICB 227 & 989.8 & NDGB 28 & 110.15 & 15.23 \\
\hline MV & 3.9 & 2.8 & CCB 4 & 6.8 & NDGB 28 & 0.7 & 17.95 \\
\hline SL (mm) & 30.65 & 24.3 & ICB 20 & 38 & CCB 4 & 2.5 & 8.14 \\
\hline UR & 50.96 & 35.8 & ICB 204 & 58.5 & ICB 124 & 4.54 & 8.92 \\
\hline BS (g/tex) & 22.83 & 18.2 & ICB 188 & 29.5 & ICB 124 & 2.92 & 12.77 \\
\hline ER & 7.3 & 4.6 & CCB 4 & 12.4 & TCB 26 & 1.31 & 17.91 \\
\hline
\end{tabular}

DTF: Days to flowering; PH: Plant height; SYM: No of sympodia; NB: No of Bolls; BW: Boll weight; SCY:

Seed cotton yield; MV: Micronnaire value; SL: Span length; UR: Uniformity ratio; BS: Bundle strength; ER:

Elongation ratio

Table.2 Eigenvalues, percentage of variability, cumulative variability and attributes that contributed towards principle components

\begin{tabular}{|l|r|r|r|r|r|}
\hline Statistics & \multicolumn{1}{|l|}{ PC1 } & PC2 & \multicolumn{1}{l|}{ PC3 } & PC4 & \multicolumn{1}{l|}{ PC5 } \\
\hline Standard deviation & 1.68 & 1.34 & 1.2 & 1.11 & 1.02 \\
\hline $\begin{array}{l}\text { Proportion of } \\
\text { Variance }\end{array}$ & 0.26 & 0.16 & 0.13 & 0.11 & 0.09 \\
\hline $\begin{array}{l}\text { Cumulative } \\
\text { Proportion }\end{array}$ & 0.26 & 0.42 & 0.55 & 0.66 & 0.76 \\
\hline Eigen Values & 2.83 & 1.8 & 1.44 & 1.23 & \multicolumn{1}{|c|}{1.03} \\
\hline Variables & PC1 & \multicolumn{1}{|c|}{ PC2 } & PC3 & PC4 & PC5 \\
\hline DTF & 0.1957 & -0.2924 & -0.2627 & 0.1246 & -0.437 \\
\hline PH & 0.1868 & -0.1041 & 0.0435 & -0.6378 & 0.3563 \\
\hline SYM & 0.3786 & 0.1054 & 0.1712 & -0.2884 & -0.1845 \\
\hline NB & 0.5072 & 0.2583 & -0.0208 & -0.0276 & -0.2213 \\
\hline BW & 0.1088 & 0.024 & 0.2281 & 0.6469 & 0.3915 \\
\hline SCY & 0.5187 & 0.2494 & 0.0533 & 0.1938 & -0.0808 \\
\hline MV & 0.3376 & -0.2223 & 0.4005 & 0.0662 & 0.068 \\
\hline SL & -0.0324 & 0.5757 & -0.2125 & -0.115 & 0.2708 \\
\hline UR & 0.2966 & -0.1125 & -0.3235 & 0.044 & 0.5836 \\
\hline BS & 0.1306 & -0.0196 & -0.7249 & 0.1246 & -0.0594 \\
\hline ER & 0.159 & -0.6076 & -0.0939 & -0.0526 & 0.1311 \\
\hline
\end{tabular}


Fig.1 Frequency distribution of 50 germplasm accessions showing different patterns of distribution

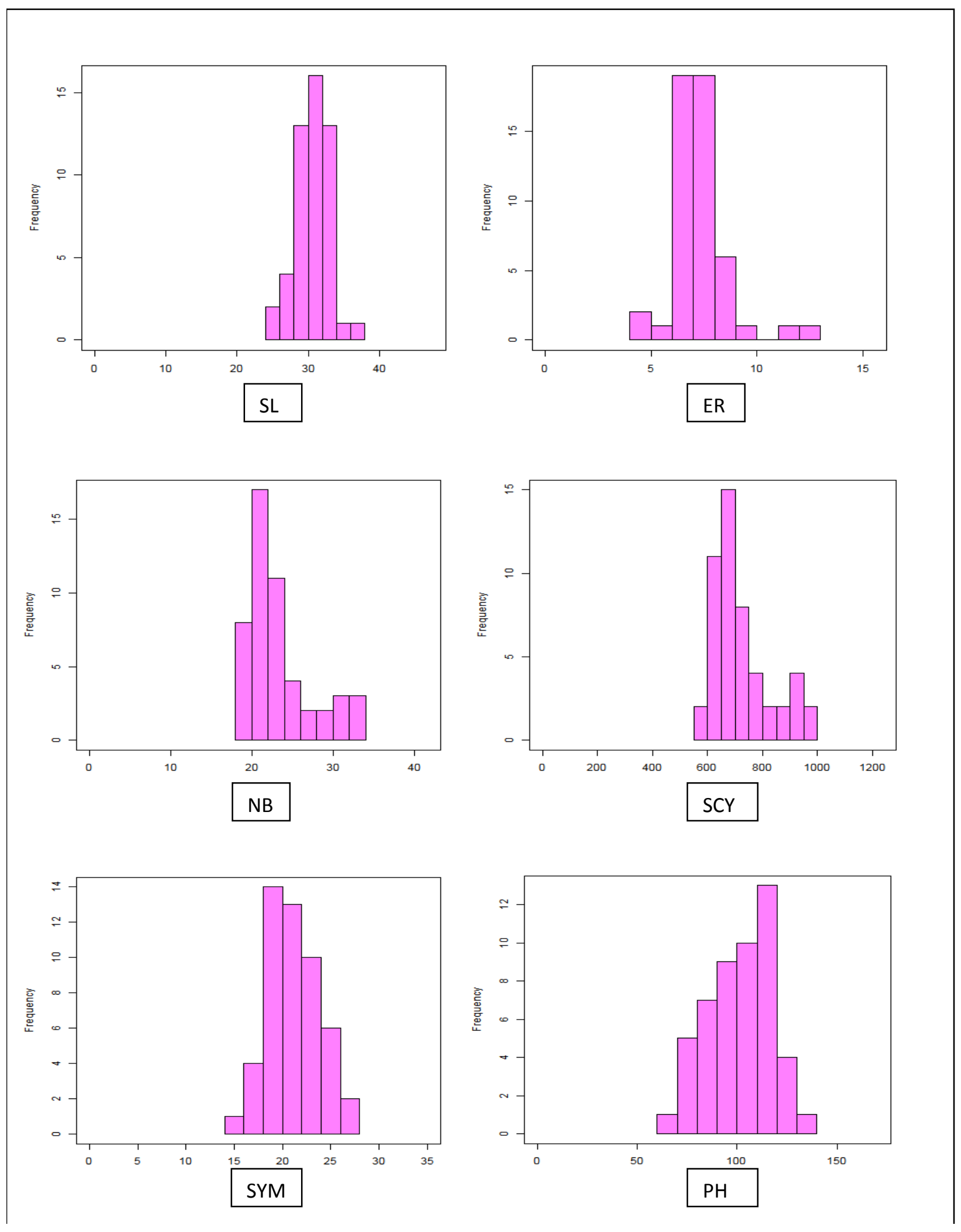


Fig.2 Biplot between PC1 and PC2 depicting the extent of variation

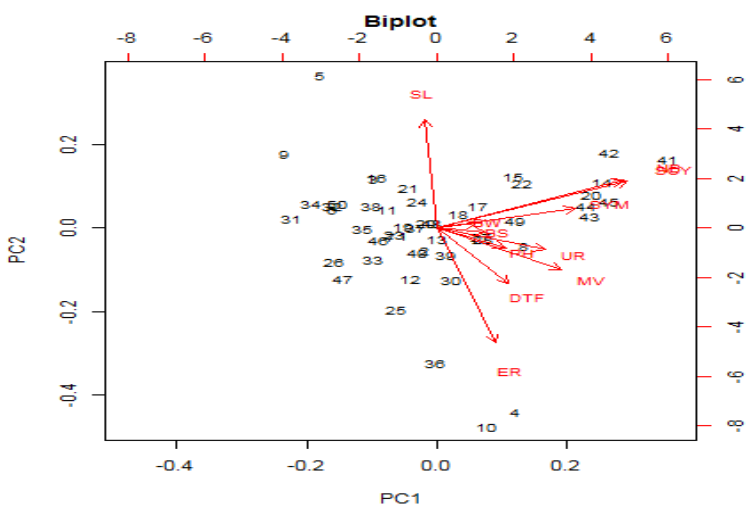

Fig.3 Score plot of fifty cotton genotypes

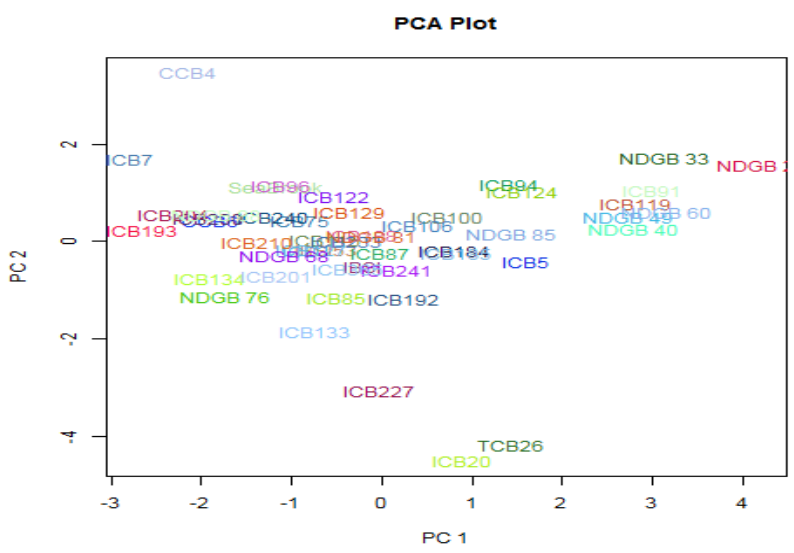

Fig.4 Scree plot showing Eigen value variation

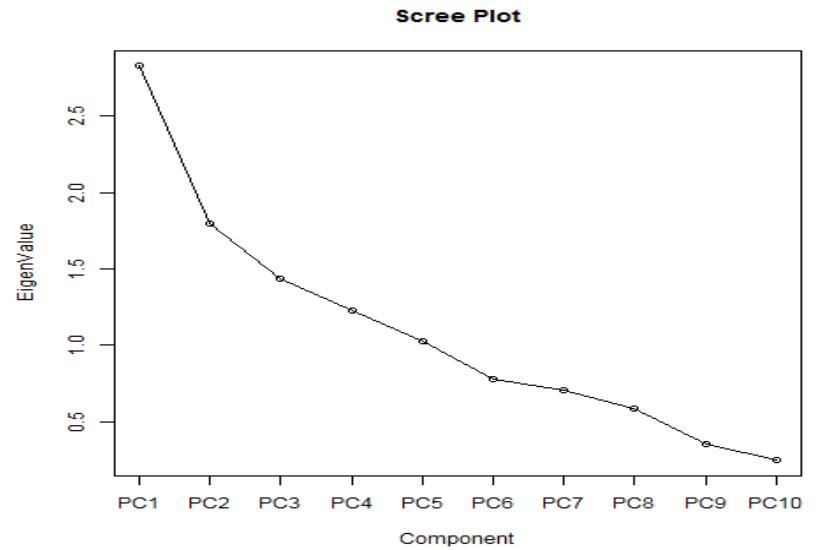

The score plot (Figure 3) scattered the germplasm based on the existence of significant genetic variation (Liaqat et al., 2015). The distance from the origin of plot and germplasm displayed the level of genetic divergence of germplasm i.e., greater distance showed significance diversity among germplasm and vice versa (Rana et al., 2013). Genetic diversity among germplasm may not rely only on their geographical distribution but also on numerous 
factors like environmental variability, exchange of hereditary material, genetic drift and natural and artificial selection (Bates et al., 1973).The results of present experiment were in greater accordance with Latif et al., (2015) and Khan et al., (2015). The score plot produced in principle components analysis diversified the fifty germplasm based on their genetic pattern (Figure 2).

The outliers ICB 20, ICB 227, TCB 26, CCB4 resulted as most divergent germplasm. On the other hand, some germplasm was present very close to the origin of polygon and supposed as being genetically similar and minimum genetic divergence due to their narrow genetic bases.

Principal component analysis has identified few characters that plays prominent role in classifying the variation existing in the germplasm set. The analysis identified seed cotton yield no of bolls, no of sympodia, boll weight, micronnaire value and span length in different principal components are the most important for classifying the variation.

Thus the results of principal component analysis used in the study have revealed the high level of genetic variation existing in the population panel and explains the traits contributing for this diversity. Hence the results will be of greater benefit to identify parents for improving various morphological traits analyzed in this study.

\section{References}

Kaleri, A.A., S.Y. Rajput, G.A. Kaleri and J.A. Marri. 2015. Analysis of Genetic diversity in genetically modified and non-modified cotton (Gossypium hirsutum L.)

\section{How to cite this article:}

Kumari Vinodhana, N. and Gunasekaran, P. 2019. Evaluation of Genetic Diversity in Cotton (Gossypium barbadense L.) Germplasm for Yield and Fibre Attributes by Principle Component Analysis. Int.J.Curr.Microbiol.App.Sci. 8(04): 2614-2621. doi: https://doi.org/10.20546/ijcmas.2019.804.304 genotypes. J. Agric.Vet. Sci. 8(12): 70-76.

Khan, F.Z., S.U. Rehman, M.A. Abid, W. Malik, C.M. Hanif, M. Bilal, G. Qanmber, A. Latif,

J. Ashraf and U. Farhan. 2015. Exploitation of germplasm for plant yield improvement in cotton (Gossypium hirsutum L.). J. Green Physiol. Genet. Genom. 1(1): 1-10.

Latif, A., M. Bilal, S.B. Hussain and F. Ahmad. 2105. Estimation of genetic divergence, association, direct and indirect effects of yield with other attributes in cotton (Gossypium hitsutum L.) using biplot correlation in and path coefficient analysis. Tropical Plant Res. 2(2): 120126.

Liaqat, S., R.I. Ahmed, S. Ahmad, M. Bilal, A. Karim, A. Qayyum, R.T. Ahmed and M. Rafiq 2015. Evaluation of diverse germplasms of cotton (Gossypium hirsutum L.) for yield and fibre attributes by multivariate analysis approach. J. Agric. Sci. Rev. 4(5): 146-150.

Rana, R.M., S. Rehman, J. Ahmad and M. Bilal. 2013. A comprehensive overview of recent advances in drought stress tolerance research in wheat (Triticum aestivum L.). Asian J. Agric.Biol. 1(1): 29-37.

Saeed, F., J. Farooq, A. Mahmood, M. Riaz, T. Hussain and A. Majeed. 2014. Assessment of genetic diversity for cotton leaf curl virus (CLCuD), fibre quality and some morphological traits using different statistical procedures in Gossypium hirsutum L. Aust. J. Crop Sci. 8(3): 442447.

Shah, A.S., Khan, J.S, Ullah.K1 and Sayal, O.U. 2018. Genetic Diversity in Cotton Germplasm using Multivariate Analysis. Sarhad Journal of Agriculture, 130-135. 